variations in elderly suicide rates, time trends in elderly suicide rates and age-associated trends in suicide rates. Psychiatric Research fournal. In press.

Shah, A. K. and Coupe, J. (2009). A comparative study of elderly suicides in England and Wales, Scotland and Northern Ireland: trends over time and age-associated trends. International Psychogeriatrics, 21, 581587.

doi:10.1017/S1041610210001559

\section{The utility of questionnaires in cognitive and functional assessment in Alzheimer's disease}

Alzheimer's disease (AD) manifests clinically with an insidious onset and slow but progressive cognitive impairment. The clinical picture of $\mathrm{AD}$ can be classified into cognitive and behavioral changes. The initial deficit usually manifests as an amnesic syndrome which may progress very gradually for several years before impairment in other cognitive domains, such as language, semantic memory and visuospatial function, becomes apparent (Hodges and Patterson, 1995).

The guidelines issued by the National Institute of Health and Clinical Excellence (2006) recommended the use of memory testing such as the Mini-mental State Examination (MMSE) and functional assessment such as the Bristol Activity of Daily Living (B-ADL) scale or the Blessed Dementia Rating Scale (BDRS) in patients with dementia.

Our aim in this study was to assess the usefulness of questionnaires in the assessment of a sample of $\mathrm{AD}$ cases referred to the memory clinic services as recommended by the NICE guidelines.

Seventy participants took part in the study. They were selected from memory clinics which met NINCDS-ADRDA criteria for mild lateonset probable $\mathrm{AD}$. Questionnaires were used to aid the diagnosis and clinical picture. The study was approved by the Local Research Ethics Committee.

The mean age of the sample was 79 years (range $=66-94, \mathrm{SD}=6.1$ ), and $61 \%$ of the subjects were female. The mean time since onset of memory problem was 1.9 years $(\mathrm{SD}=.97)$. Seventeen percent $(\mathrm{N}=12)$ of subjects used a hearing aid and $8 \%(\mathrm{~N}=6)$ wore spectacles. All subjects were mobile with no abnormalities.

All subjects scored 0 on the Neuropsychiatric Inventory and Webster Extrapyramidal Symptoms scales, excluding any other pathology such as Parkinson's disease, dementia with Lewy bodies and vascular brain incidents. All subjects scored 0-1 on

\begin{abstract}
AJIT SHAH
Professor of Ageing, Ethnicity and Mental Health, International School for Communities, Rights and Inclusion, University of Central Lancashire, Preston, U.K. and Consultant Psychiatrist, West London Mental Health NHS Trust, London, U.K. Email: ajitshah123@btinternet.com
\end{abstract}

the Geriatric Depression Scale, which excluded the presence of depression.

The MMSE was used to screen the cognitive functions of all participants. The mean MMSE score was 23.30 (range $=19-26, \mathrm{SD}=2.87$ ). Mann-Whitney $U$ test was used to test the relationship between gender and MMSE and it showed no significant difference $(\mathrm{U}=36.00$, $p>0.05)$. Multiple regression analysis showed no correlation between age and MMSE $(F=-0.116$, $t(68)=-0.961, p>0.05)$.

The mean score for total Cambridge Mental Disorders of the Elderly Examination (CAMDEX) was $1.30(\mathrm{SD}=0.66) \cdot \chi^{2}$ test by gender and CAMDEX showed no significant difference $\left(\chi^{2}(1\right.$, $70)=3.657, p>0.05$. The mean B-ADL score was $2.40(\mathrm{SD}=1.31) \cdot \chi^{2}$ test showed no significant relationship between B-ADL and gender $\left(\chi^{2}\right.$ $(1,70)=3.200, p>0.05$.

Correlation analysis shows a significant negative correlation between MMSE, and years since onset, $\mathrm{r}=-0.444, p<0.05$. A highly significant positive correlation was found between year since onset of dementia and total B-ADL $(\mathrm{r}=0.778$, $p<0.01)$. There is also a highly significant correlation between total CAMDEX and the BDRS $(\mathrm{r}=0.681, p<0.01)$. Repeated measure ANOVA showed no significant main effect for age. However, there was a significant relationship between gender and B-ADL $(F(1,1)=6.293, p<0.05)$ and gender and total CAMDEX $(F(1,1)=4.702$, $p<0.05)$.

Cognitive, psychiatric and functional assessment has important implications for the management of $\mathrm{AD}$ including planning for services and the use of cholinesterase inhibitors. The five areas that require assessment (and periodic reassessment) in a patient with $\mathrm{AD}$ are daily function, cognition, comorbid medical conditions, and disorders of mood and emotion, and caregiver status (Cummings et al., 2002).

In our study, correlation analysis shows a significant correlation between years since onset of memory problem and both MMSE, and total BADL. This means that the longer subjects have had the illness and the severer the cognitive decline, the 
more impairment they present with. Our sample did not show any other significant psychopathology, such as psychosis or affective disorder. Because of the mild degree of cognitive impairment and early $\mathrm{AD}$, functional assessment revealed normal activities. However, the correlation between total CAMDEX and BDRS reflecting the general decline in functioning associated with dementia.

Some of the questionnaires used were selfrated while others were carer-rated. Koss et al. (1993) found that caregivers of patients with $\mathrm{AD}$ were reliable informants of their relatives' deficits. Holmes and Lovestone (2003) concluded that in the evaluation of individual treatment response, the rate of change - as measured by the MMSE and BDRS is of limited value. Moreover, Herrmann (1982) found that responses to memory questionnaires varied with several variables: the type of memory failure, susceptibility to cognitive failures under stress, confidence in memory performance, and age of subjects.

Questionnaires are used as a part of clinical evaluation of cognitive and functional impairment. Assessment also includes history taking, examination, and blood and radiological investigations. Regular reassessments are important to all aspects of the management of $\mathrm{AD}$ patients and questionnaires can assist in tracking change over time. Another important advantage of using questionnaires is the determination of the patient's capacity to engage in the assessment and the care plan that will ensure the delivery of appropriate care as early as possible. Factors to take into account in using different questionnaires include the questionnaire itself and its specificity, validity, negative and positive predictive values; the raters' variability in scoring; and the individual differences in cognitive and functional abilities. We conclude that questionnaires are important tools in the care of patients with dementia in general and $\mathrm{AD}$ in particular.

\section{References}

Cummings, J. L. et al. (2002). Guidelines for managing Alzheimer's disease: Part I. Assessment. American Family Physician, 65, 2263-2272.

Herrmann, D. (1982). Know the memory: the use of questionnaires to assess and study memory. Psychological Bulletin, 92, 434-452.

Hodges, J. R. and Patterson, K. (1995). Is semantic memory consistently impaired early in the course of Alzheimer's disease? Neuroanatomical and diagnostic implications. Neuropsychologia, 33, 441-459.

Holmes, C. and Lovestone, S. (2003). Long-term cognitive and functional decline in late onset Alzheimer's disease: therapeutic implications. Age and Ageing, 32, 200-204.

Koss, E., Patterson, M. B., Ownby, R., Stuckey, J. C. and Whitehouse, P. J. (1993). Memory evaluation in Alzheimer's disease caregivers' appraisals and objective testing. Archives of Neurology, 50, 92-97.

National Institute of Health and Clinical Excellence (2006). Dementia: Supporting People with Dementia and their Carers in Health and Social Care. NICE Clinical Guideline 42. London: NICE.

V M AzIZ, ${ }^{1}$ J. Yagoub, ${ }^{2}$ K. SABA ${ }^{3}$ AND M. $\operatorname{ASAAD}^{4}$

${ }^{1}$ Consultant psychiatrist, Cwm Taf LHB. St Tydfil's Hospital, Merthyr Tydfil, Wales. U.K.

${ }^{2}$ Medical student, St George's Medical School, London, U.K.

${ }^{3}$ Medical student, University of Cardiff Medical School, Cardiff, Wales, U.K.

${ }^{4}$ Medical student, Oxford University Medical School, Oxford, U.K.

Email: victoraziz@doctors.org.uk 